\title{
Factors influencing antibiotic prescribing in China: An exploratory analysis
}

\section{Lucy Reynolds, Martin McKee *}

European Centre on Health of Societies in Transition, London School of Hygiene and Tropical Medicine, Keppel St re et, London WC1E 7HT, United Kingdom

\author{
abstract \\ Objectives: China has very high rates of antibiotic resistance and a health care system that provides strong incentives for over-prescribing. This paper \\ describes the findings of a qualitative study in a province of southern China that seeks to assess knowledge, attitudes, and practices in relation to the
} use of antibiotics.

Methods: Semi-structured interviews with patients and health workers at provincial, county, township, and village level. Interviews used four probes (common cold, cough, mild diarrhoea and tiredness) where antibiotics were not indicated, supplemented by questions on knowledge, attitudes, and practices. These data were supplemented by two focus groups, with medical students and pharmacists, and discussions with participants at a national conference on antibiotic use.

Results: Coughs and diarrhoea are almost universally treated with antibiotics, while the cold is normally treated with antivirals instead or as well. Many physicians are aware that the cold is usually self-limiting but believe that they can speed recovery and that they are responding to patient expectations. Most physicians and many patients are aware of the phenomenon of antibiotic resistance, although it is often seen as a property acquired by the patient and not the micro-organism. Physicians face financial incentives to prescribe, with profit splitting with pharmaceutical suppliers. Sales profits form a major part of a hospital's income. National guidance on use of antibiotics is fragmentary and incomplete.

Conclusion: The misuse of antibiotics poses considerable risks. Effective action will require a multi-faceted strategy including education, based on an understanding of existing beliefs, the replacement of perverse incentives with those promoting best practice, and investment in improved surveillance. Much of this will require action at national level.

\section{Introduction}

Rates of antibiotic resistance in China are growing rapidly from already high levels [1-4]. At least part of the explanation lies in changes in the health system over the past two decades. The health system that China established in the 1950 s was a remarkable improvement on what had existed previously. Medical training and hospital construction increased greatly, while effective campaigns were conducted against endemic diseases such as schistosomiasis and malaria. Primary health care provision in rural areas was boosted by the introduction of "barefoot doctors". These changes made it possible for $90 \%$ of the population to access at least basic care [5] and contributed to China's marked improvement in life expectancy in the three decades following the Communist takeover, from 35 years in 1949 to 68 years in 1981 [6]. Nevertheless, by 1981, healthcare accounted for only $3 \%$ of a comparatively low GDP [7]

During the reforms instituted by Deng Xiaoping in the early 1980s, the health system deteriorated, suffering from low salaries and a lack of investment [8]. The introduction of "market socialism" in all aspects of public life led to further reductions in resources. A new system, introduced in the early 1990s [9], limited state financing to about 25-30\% of total costs [10], covering only the basic costs of salaries and facilities. Staff were to supplement their income from bonus schemes, funded from sales profits on drugs, tests and services [9] with staff fined for missing sales quotas [11]. Medical insurance covered those employed in state enterprises, but with a decreasing proportion of the population in such employment, by 2005 only 25\% of people living in urban areas [12] and 10\% of rural Chinese had any cover [7]. Costs of insurance schemes escalated, as prices rose to cover profit-loading by providers, and over-prescription escalated claims [13], so cost-sharing and claims ceilings were introduced to limit expenditure [9].

Studies carried out in the late 1990s provided evidence that fee-for-service provision, provider bonuses, and medical insurance all encouraged prescription of more items, more expensive medications, and newer antibiotics [5,14-16]. The result was that per capita consumption of medicines increased almost fourfold between 1979 and 1992 [8]. By the mid-1990s, more than half of hospital spending was on pharmaceuticals $[5,7,10,17]$, with one survey in 1990 finding that each visit to a physician led to the prescription, on average, of 2.3 drugs [18] while another, among villages in western China found that about half of all prescriptions were for antibiotics [19]. Liu and Mills reported over-prescribing (expressed as cost of drugs) of 38\% for pneumonia and 34\% for appendicitis in six public hospitals in the period 1987-1997 [20], while Meng et al. [21], in a 2000 study in 109 village clinics in Shandong province, found over-treatment (again by cost) of $60 \%$. A study reported in 2005, from Beijing found overuse of broadspectrum antibiotics, especially the most expensive [22]. Interviews and focus groups attributed this to the policy of requiring hospitals to support themselves from profits, gaps in the knowledge of junior staff coupled with a tendency to rely on information from pharmaceutical companies, and concern about possible litigation. 
problem was due, primarily, to "the over-the-counter purchase and community use of fluoroquinolines in the People's Republic of China" [24].

While the scale and nature of the problem are apparent, there is rather less research on how the system shapes decisions made by patients and physicians in their everyday encounters. This information will be essential if effective policies are to be put in place. In this paper, we report a qualitative investigation of the determinants of prescribing for common conditions in one province of southern China.

\section{Methods}

The research was set in Guizhou, a landlocked province in southern China, bordered by Sichuan to the north, Yunnan to the west, Guangxi to the south and Hunan to the east. Much of the province is mountainous, with forestry and coal mining dominating the economy. Almost $40 \%$ of the population are from China's minority ethnic groups. This study was part of a larger one looking at the entire range of iatrogenic factors that might play a role in transmission of blood-borne viruses. The other elements will be described elsewhere [25].

The approach was based on grounded theory, whereby themes emerge from initial analyses which are then explored in an iterative manner through purposive sampling of relevant key informants and triangulation with other sources of data [26]. The main source of data was a series of semi-structured interviews conducted in rural and urban Guizhou with a purposive sample of healthcare staff, patients, and other key informants, including civil representatives, at provincial, county, and village facilities. Three villages were selected, in consultation with the provincial staff of the Chinese Centres for Disease Control (CDC), with the goal of including ones with different levels of economic development

Patients were identified by convenience sampling from those present in the health facility on the selected days. The health workers interviewed were, at village level, doctors, and at township, county and provincial level, the hospital director, surgeon/ obstetrician, and officials in charge of safety and procurement. Additional key informants included village leaders, family planning officials, and state and independent pharmacists.

The interview framework was structured around four different minor tracer complaints, common cold, cough, mild diarrhoea and tiredness, chosen as ailments that would rarely merit any medical intervention if rational treatment principles were followed. Follow up questions pursued points raised by interviewees about influences on prescription choices. The interviews were all carried out by LR, in the presence of a local research assistant who was fluent in the local dialect. Immediately after each interview, both checked transcripts together to resolve any ambiguities. The transcriptions were then read and analysed by both authors to identify emerging themes. These themes were then explored in more detail with key informants with knowledge of the Chinese health system and in two focus groups. One comprised university students, addressing the impact of pharmaceutical advertising, and the other comprised pharmacists in the Guizhou provincial hospital, to explore procedural and legal constraints on prescribing. In both cases a list of relevant topics was derived from the interviews and participants were presented with corresponding scenarios. LR led the focus groups, which were then transcribed by a research assistant. Less formal individual and group interviews were conducted with clinicians and researchers at the December 2005 Second National Conference on Rational Use of Antibiotics in Beijing. This was designed to provide some idea of whether the findings obtained were exceptional in China or whether they could, with caution, be generalised.

Ethical approval was granted by the relevant committees of the Chinese Ministry of Health and the London School of Hygiene and Tropical Medicine.

\section{Results}

The initial sample comprise d 24 patients ( 15 rural, 9 urban), 11 village doctors, health workers at higher levels of the system (township hospital, 4; county hospital, 7; provincial hospital, 15), two independent pharmacists, 2 village leaders, and 3 family planning officials. Subsequent interviews, to clarify emerging themes, were conducted with two other doctors in the state system two in the private sector, and two in non-governmental organisations, as well as three from the Centre for Disease Control and three 3 foreign health workers. The last group comprised North Americans who had been working with different non-governmental organisations in China for bet ween 12 and 20 years and who therefore were i n a position to provide an international perspective.

\subsection{Antibiotic treatment by clinicians}

Healthcare workers and key informants reported that antibiotics are not only used incorrectly for susceptible bacterial infections, but also frequently for problems known not to have bacterial aetiology. Those suffering from any complaint other than tiredness routinely received antibiotic treatment. Many, but not all healthcare workers, were aware that a cold is a self-limiting viral infection, but most treated it with antivirals (ribavirin, acyclovir or moroxydine), antibiotics, or both, sometimes combined with symptomatic treatments and Traditional Chinese Medicines (TCM). Because colds are self-resolving, the healthcare worker who treats them benefits from a $\mathrm{n}$ apparently successful cure, promoting recommendations by patients and leading to a cycle of over-treatment. Some prescribers did disapprove of using antibiotics for colds; one outpatient doctor reported refusing to prescribe them unless pressurise $\mathrm{d}$ by patients. Coughs were also treated with antibiotics at all levels of the system. 
For mild diarrhoea, quinolines and sulphonamides are prescribed. At the provincial hospital, treatment is usually with intravenous (IV) antibiotics selected according to the results of stool tests when available, but sometimes blindly.

\subsection{Perceptions of antibiotic resistance}

Awareness of the phenomenon of antibiotic resistance was generally high, even if not always fully understood. A doctor in the county hospital reported that penicillin work s less well for city people than for villagers, whose access to care is limited by their low incomes, indicating an understanding that its development was linked to previous use. Both healthcare workers and patients had notice $d$ that antibiotics were gradually becoming less effective. However, this was of ten attributed to the patient developing resistance rather than the micro-organism. One patient observe $d$ that "if you take injections and infusions easily, you can damage your immunity and will become resistant to medicine". An older hospital pharmacist commented

"antibiotic dosages have risen greatly. This is because people have some substance in their bodies which can resist the drug; if they can be treated by one pill the first time, the second time it takes two pills and the third time three pills."

\subsection{Initiatives to reduce misuse of antibiotics}

Diagnosis is most often based on symptoms. Laboratory confirmation and testing for antibiotic sensitivity are rare, reflecting both lack of access to laboratory facilities in villages and affordability in cities. Clinicians typically try different antibiotics until symptoms cease.

Many doctors interviewed expressed concern about the potential ill effects of antibiotic use; yet most, by their own accounts, considerably over-prescribed. Their concern was mainly to avoid long courses, to which they attributed toxicity, damage to microflora and the development of resistance. No respondent mentioned inappropriate use of broad-spectrum or unsuitable antibiotics. Few were aware that courses that were too short might facilitate the emergence of resistant strains.

In autumn 2004, a nationwide guideline was issued to hospitals, designating three classes of antibiotics based on clinical and safety profiles, with different rules for prescribing each class [27]. As explained by a provincial hospital doctor "Ordinary doctors in the hospital can prescribe the common antibiotics such as penicillin, but for the higher level antibiotics, they must seek permission from their head of department. For the new and expensive antibiotics, as we are not yet sure of the clinical implications, they must consult the specialists."

Younger doctors seemed more likely to start with the newest antibiotics, for profit or to demonstrate swift effect, but most follow the guidance to start with penicillin, despite widespread beta-lactam resistance. This leads to the inappropriate use of high dosages, in the hope of observing some effect. A pharmacist commented: "In the 1960s, the highest dosage of penicillin was 400,000 i.u., but now it is up to $800,000-1,200,000$ i.u. In the past a cure required four shots, but now six to eight are needed." While penicillin is relatively non-toxic at high doses, the idea of increasing dosages is carried across to other antibiotics where the hazards are greater. In this way, well-intentioned official guidance may be having perverse effects.

\subsection{Economic influences on antibiotic prescription: customers and suppliers}

Health workers recognised the financial incentives to prescribe as taking two forms. One was created by pharmaceutical companies, who sometimes arrange to split profits with prescribers, illegally and covertly, while the other emanated from all health providers, which are entitled to make a $15 \%$ profit on sales to fund services, but often inflate invoices to suppliers to increase the absolute profit. Some doctors did deplore the phenomenon of profit-led prescription but it was generally accepted as inevitable. It was noted that patients want their symptoms alleviated as quickly as possible, while minimising the fees they pay. Healthcare workers aim to maximise these fees, but share patients' interest in fast treatment, since this attracts and retains patients. One consequence of this dynamic is over-prescription of antibiotics, especially the newest ones. Another is the prescription of only enough antibiotics to clear the patient's symptoms, since the patient does not comprehend the benefit of paying for the rest of the course.

Doctors and researchers attending the conference on antibiotic use confirmed the barriers to rational prescribing imposed by current hospital funding arrangements, which rely on maximising drug sales profits. They asserted that a shift to rational prescribing would destroy the revenue base of hospitals, further degrading service quality because of the cost-cutting necessary to break even without the income earned from irrational use of medication.

3.5. Use of over-the-counter antibiotics

Patients have acquired the habit of self-medication. A County Hospital outpatient doctor explained:

"Many patients abuse antibiotics before they come to hospital, and have already developed drug resistance before they arrive. Thus each antibiotic course is 3 days, but the overall treatment is usually of longer duration." 
remedy. Rural interviewees mentioned purchase of over-the-counter norfloxacin, furazolidine and cotrimoxazole for diarrhoea, and sulphanilamide to treat colds. Maintaining home stocks of antibiotics for family use is reportedly normal, as is taking antibiotics for diarrhoea several times a year.

On 1 July 2004, the State Food and Drug Administration announced that pharmacies would no longer sell antibiotics without a prescription [28]. Interviews and observation at two pharmacies (one large and one small) indicated that the letter of the law was observed. However, the law's spirit can be circumvented; staff at the large pharmacy explained that a doctor works there every morning who can prescribe as required. A researcher interviewed at the national conference confirmed the ubiquity of this practice and commented that the patient need not even be present at the pharmacy.

\subsection{Sources of guidance on antibiotic prescription}

Healthcare workers were asked what authorities they consult in decisions about prescribing. Existing guidance on antibiotic use appears to be profuse but inadequate. Clinicians referred to the frequency with which national guidance was issued, with no single authoritative documentary source available. No actual clinical guidelines could be identified at any level of the system, from the village to the provincial hospital.

Some of the misconceptions about antibiotic use encountered were widely shared. A possible mechanism for their propagation and reinforcement is the acquisition of incorrect knowledge in teaching hospitals, which is then disseminated more widely. One doctor mentioned the Ministry of Health website (http://www.moh.gov.cn) as a source of clinical guidance on antibiotic use; this stated

"70\% of prescriptions are for antibiotics; China is now one of the heaviest users in the world. Among China's 5 million tuberculosis patients, at least a quarter carry bacteria resistant to at least one antibiotic. Now people have realised the danger of antibiotic abuse. As a result we should strengthen rational prescription to stop antibiotic abuse, to reduce the side-effects and the costs caused by irrational prescription... . misuse will cause drug resistance".

However, this website gives no explanation about how to decide when to stop antibiotic courses, what rational prescription of antibiotics requires, or what "misuse" entails.

When infections fail to resolve, patients are typically prescribed a further course but of different antibiotics. Thus, a single infection will often lead to multiple 3-day courses of different antimicrobials, often commencing with over the counter treatments. Wellintended but unclear government publicity about antibiotic abuse may have increased the frequency of prescriptions for short courses of antibiotics, exacerbating resistance.

\section{Discussion}

This study has a number of obvious limitations. It was undertaken in a single province of a vast and extremely diverse country and the number of interviewees was relatively small. There is a clear need to replicate this work in other parts of China. However, the findings were discussed with participants at a national conference, eliciting a consensus that the findings were not atypical.

The time required to conduct semi-structured interviews limited the numbers that could be included but also had numerous advantages. These included the ability to probe responses fully to clarify any ambiguities, to obtain information of greater depth, and to resolve misunderstandings, while ensuring a high response rate [29].

The findings are of considerable concern. They reveal widespread prescribing of unnecessary antibiotics, often administered parenterally. This is dangerous for two reasons. First, it encourages the development of antibiotic resistance, a phenomenon that, as we show, is imperfectly understood. Second, the use of injections and infusions poses a potential threat from spread of blood-borne viruses and infusion site infections. This study also sheds light on the reasons for this situation, including the existence of financial incentives and the limited availability of information on appropriate prescribing. The widespread use of anti-virals for self-limiting illness is especially striking, and is quite at variance with accepted practice elsewhere.

A number of obvious implications flow from the findings. Bringing about behavioural change, especially when it is deeply entrenched, is never easy. However, some principles that should inform any policy can be discerned. First, there is a need for a multi-faceted strategy that encompasses education, regulation, and creation of incentives. Educational approaches should begin from what people already believe. As this study has shown, the concept of antibiotic resistance is known but imperfectly understood. An educational programme must begin by conveying knowledge that it is the micro-organism that develops resistance and not the patient. It should then convey at least a basic understanding of the evolutionary pressures that lead resistance to emerge. Learning should stress the need for sensitivity testing before switching antibiotics when treatment fails. Educational strategies could usefully be complemented by enhanced feedback, based on surveillance of emerging patterns of resistance, linked to guidance on the most appropriate antibiotics for each situation. Drug utilisation reviews in hospitals, as recommended by the WHO [30], can also play a part. China does have an essential drugs list but there is a need for greater awareness and use of it. 
Yet education alone will not be enough, a view confirmed by an experience of applying Australian guidelines in Chinese hospitals [31]. As that study and ours found, there is a fundamental need to address the perverse economic incentives that encourage inappropriate prescribing. It is entirely understandable that physicians will overprescribe when they see this as the only way of ensuring the viability of their facilities. There is a need to abolish the $15 \%$ cap on profits that paradoxically increases both the unit price paid and volume of unnecessary antibiotics prescribed. Other areas demanding attention are the bonuses paid by pharmaceutical companies to hospitals and by hospitals to prescribers in return for meeting sales targets.

Stepping back a little, it is apparent that the function identified by the WHO as stewardship, defined as the 'the careful and responsible management of the well-being of the population', is lacking [32]. This function resides in different places in health systems but those organisations that co-pay for care are well placed to exercise it. In China, the fragmentation of the insurance sector, with a multitude of small schemes, poses an obstacle to assuming this role. While it is not necessary to have structural consolidation of this sector (though this would be one solution, following the example of the Australian Pharmaceutical Benefits Scheme), more could be done through other mechanisms to collaborate in the development, implementation, and monitoring of evidence-based prescribing. What is important is that someone, somewhere, takes charge of addressing the quality of medicines provision.

\section{References}

[1] Zhang R, et al. Antibiotic resistance as a global threat: evidence from China, Kuwait and the United States. Global Health 2006;2:p6.

[2] Yao KH, Yang YH. Streptococcus pneumoniae diseases in Chinese children: past, present and future. Vaccine 2008;26(35):4425-33.

[3] Li J, Ma Y, Wang Z, Yu X. Klebsiella pneumoniae: epidemiology and analysis of risk factors for infections caused by resistant strains. Chinese Medical Journal (English) 2002;115(8):1158-62.

[4] Shen D, Biedenbach DJ, Winokur PL, Pfaller MA, Jones RN. Phenotypic and genotypic characterizations of Chinese strains of Escherichia coli producing extended-spectrum beta-lactamases. Diagnostic Microbiology and Infectious Disease 1999;34(3):159-64.

[5] Bogg L, Dong H, Wang K, Cai W, Vinod D. The cost of coverage: rural health insurance in China. Health Policy Plan 1996;11(3):238-52.

[6] Xu W. Flourishing health work in China. Social Science and Medicine 1995;41(8):1043-5.

[7] World Bank. Financing health care: issues and options for China. Washington, DC: World Bank; 1997.

[8] Liu X, Li S. Drug policy in China. Transformations, current status and future prospects. Pharmacoeconomics 1997;12(1):1-9.

[9] Lok SH. Market reform and China's health care system. Social Science and Medicine 1995;41(8):1065-72.

[10] Liu X, Liu Y, Chen N. The Chinese experience of hospital price regulation. Health Policy Plan 2000;15(2):157-63.

[11] Zheng X, Hillier S. The reforms of the Chinese health care system: county level changes: the Jiangxi study. Social Science and Medicine 1995;41(8):1057-64.

[12] Ministry of Health. Report on China's Healthcare System and Reform. Beijing: Ministry of Health; 2005.

[13] Bloom G, Lucas H, Cao SH, Gao JM, Yun J, Gu XY. Financing health services in poor rural areas: adapting to economic and institutional reform in China. Research Report 30. Brighton: Institute of Development Studies, Sussex University; 1995.

[14] Dong H, Bogg L, Wang K, Rehnberg C, Diwan V. A description of outpatient drug use in rural China: evidence of differences due to insurance coverage. International Journal of Health Planning and Management 1999;14(1):41-56.

[15] Dong HL, Bogg L, Rehnberg C, Diwan V. Health Financing Policies: providers opinions and prescribing behaviour in rural China. International Journal of Technology Assessment in Health Care 1999;15(4):686-98.

[16] Dong H, Bogg L, Rehnberg C, Diwan V. Association between health insurance and antibiotics prescribing in four counties in rural China. Health Policy 1999;48(1):29-45.

[17] Wei Y. Memo on revised National Health Accounts for 1993. Washington, DC: World Bank; 1996.

[18] Banta HD. Medical technology in China. Health Policy 1990;14:127-37.

[19] Dong L, Yan H, Wang D. Antibiotic prescribing patterns in village health clinics across 10 provinces of Western China. Journal of Antimicrobial Chemotherapy 2008.

[20] Liu X, Mills A. Evaluating payment mechanisms: how can we measure unnecessary care? Health Policy Plan 1999;14(4):409-13.

[21] Meng Q, Liu X, Shi J. Comparing the services and quality of private and public clinics in rural China. Health Policy Plan 2000;15(4):349-56.

[22] Zhang Y. Improving prophylactic antibiotic prescribing for surgeons in a general hospital in Beijing - lessons from a trial of localised antibiotic guidelines, drug utilisation study and education. In: Second National Workshop on Rational Use of Antibiotics in China. 2005.

[23] Hu S, Liu X, Peng Y. Assessment of antibiotic prescription in hospitalised patients at a Chinese university hospital. Journal of Infection 2003;46(3):161-3.

[24] Ling TK, et al. Multicenter antimicrobial susceptibility survey of gram-negative bacteria isolated from patients with communityacquired infections in the People's Republic of China. Antimicrobial Agents and Chemotherapy 2006;50(1):374-8.

[25] Reynolds, L, McKee, M. Possible risks of transmission of blood-borne infection via acupuncture needles in Guizhou province of South-West China. Journal of Alternative and Complementary Medicine; in press.

[26] Glaser B, Strauss A. The discovery of grounded theory: strategies for qualitative research. Chicago: Aldine; 1967.

[27] Xinhua News Agency. First Guideline on Clinical Use of Antibiotics Issued 2004 [cited 12th April 2008]; Available from: http://www. china.org.cn/english/2004/Oct/108983.htm.

[28] Lu, E. Strengthening Control of Antibiotics. 2005 [cited 12 th April 2008]; Available from:

http://www.chinatoday.com.cn/English/e2005/e200503/p40.htm.

[29] Bowling A. Research methods in health: investigating health and health services. Buckingham: Open University Press; 1997.

[30] Avorn JL, Barrett JF, Davey PG, McEwen SA, O’Brien TF, Levy SB. Antibiotic Resistance: synthesis of recommendations by expert policy groups. In: Alliance for the prudent use of antibiotics. Geneva: World Health Organization; 2001.

[31] Zhang Y, Harvey K. Rational antibiotic use in China: lessons learnt through introducing surgeons to Australian guidelines. Australian and New Zealand Health Policy 2006;3:p5.

[32] Saltman RB, Ferroussier-Davis 0. The concept of stewardship in health policy. Bulletin of the World Health Organization 2000;78(6):732-9. 